\title{
MULTI-SCALE FULL-FIELD MEASUREMENTS AND NEAR-WALL MODELING OF TURBULENT SUBCOOLED BOILING FLOW USING INNOVATIVE EXPERIMENTAL TECHNIQUES
}

\author{
Yassin A. Hassan \\ Texas A\&M University - Nuclear Engineering Department \\ College Station, TX, 77843-3133 \\ Email: y-hassan@tamu.edu
}

\begin{abstract}
Multi-phase flows are one of the challenges on which the CFD simulation community has been working extensively with a relatively low success. The phenomena associated behind the momentum and heat transfer mechanisms associated to multi-phase flows are highly complex requiring resolving simultaneously for multiple scales on time and space. Part of the reasons behind the low predictive capability of CFD when studying multi-phase flows, is the scarcity of CFD-grade experimental data for validation. The complexity of the phenomena and its sensitivity to small sources of perturbations makes its measurements a difficult task. Non-intrusive and innovative measuring techniques are required to accurately measure multi-phase flow parameters while at the same time satisfying the high resolution required to validate CFD simulations. In this context, this work explores the feasible implementation of innovative measuring techniques that can provide whole-field and multi-scale measurements of two-phase flow turbulence, heat transfer, and boiling parameters. To this end, three visualization techniques are simultaneously implemented to study subcooled boiling flow through a vertical rectangular channel with a single heated wall. These techniques are listed next and are used as follow: 1) High-speed infrared thermometry (IR-T) is used to study the impact of the boiling level on the heat transfer coefficients at the heated wall, 2) Particle Tracking Velocimetry (PTV) is used to analyze the influence that boiling parameters have on the liquid phase turbulence statistics, 3) Highspeed shadowgraphy with LED illumination is used to obtain the gas phase dynamics. To account for the accuracy and to complement these innovative techniques, redundant and simultaneous measurements are performed by means of thermocouples, flow and power meters, differential and absolute pressure transducers, etc. The present experiments are intended to improve the understanding of subcooled boiling flow and to provide reliable and accurate subcooled boiling flow experimental information for verification and validation of two-phase flow computational models.
\end{abstract}

Keywords: Subcooled boiling, particle image velocimetry, infrared thermometry, shadowgraphy images, two-phase flows

\section{INTRODUCTION}

Turbulent subcooled boiling flow has been used extensively in industry because it is one of the most efficient heat transfer modes. The continuous mixing and stirring of liquid produced during the life cycle of the subcooled boiling bubbles due to nucleation, growth, detachment, coalescence and collapsing are enhancing mechanisms of heat and momentum transfer. The understanding of twophase flow parameters and their impact on the liquid turbulence plays a key role on the behaviour prediction, safety analysis and design of the high energy systems found in industry.

In particular, the nuclear industry relies on the accurate prediction of local two-phase flow parameters. For example, the nucleation sites location and the amount of void within coolant/moderator subchannels affect not only the reactor's reactivity, but also promote the crud formation which has been 
shown to contribute to the generation of heat transfer non-uniformities along the fuel rods and ultimately fuel rod structural failures. Although multiple experimental efforts has been directed towards the understanding of subcooled boiling flow, the complexity of the phenomenon and its high dependence on the experimental conditions has produced large discrepancies among experimental results, even at similar conditions.

Therefore there is a need of innovative experimental techniques that can fulfill the demanding statistical requirements to describe the stochastic nature of subcooled flow boiling. This work explores the scope and limitations of innovative measuring techniques to study subcooled boiling flow. Efforts for the simultaneous measurements of liquid and vapor parameters and their interactions are described. Emphasis is given to achieve measurements with the required temporal and spatial resolutions for both: average and fluctuating quantities. There are multiple studies on subcooled boiling flow, but for sake of brevity, only those relevant to this study are classified in the following groups: 1) studies that focus on measuring average turbulence quantities and 2) studies that focus on the measurement of bubbles dynamics parameters. Most of the studies that focus on measuring average turbulence quantities rely on point measurement probes and generally focus on the study of the liquid turbulence and local void properties. One of the early attempts to measure local fields of subcooled boiling parameters of the liquid phase was performed by Roy et al. (1993). They measured turbulent velocity and temperature fields in the all-liquid region adjacent to a subcooled flow boiling layer. Significant changes in the turbulent structure of the all-liquid region were observed due to boiling. Improving their measurement techniques, Roy et al. (1997) were able to measure liquid turbulence statistics of the liquid refrigerant R-113 even within the boiling layer region adjacent to the heated wall. They found that the near-wall liquid velocity field was significantly different from that in single-phase liquid flow at a similar Reynolds number. Lee et al. (2002a) performed measurements of subcooled boiling flow of water in a vertical concentric annulus. Using a two-conductivity probe they measured the local void fraction and vapour velocity and with a Pitot tube they measured the liquid velocity. Situ et al. (2004) measured the flow structure of subcooled boiling flow in an annulus. They used a double-sensor conductivity probe method to measure local void fraction, interfacial area concentration and interfacial velocities. Using the experimental information from the previous studies, different two-phase flow models were developed and used to calculate convective subcooled boiling flow (Končar et al. 2004; Koncar et al. 2005; Koncar \& Matkovic 2012; Koncar \& Tiselj 2010; Ramstorfer et al. 2008) with some success. These models share the characteristics of being based on time-average analysis of information from point measurements probes. However, due to the complex nature of the turbulence found in subcooled boiling, this approach seems to be limited. A full-field measurement approach is needed to provide spatial and temporal information. Visualization techniques such as Particle Image Velocimetry (PIV) and Particle Tacking Velocimetry (PTV) can be used to overcome some of the limitations associated with point measurements techniques. Using visualization techniques in conjunction with PTV, several adiabatic two-phase flow experimental studies were performed (E.E. Dominguez-Ontiveros et al. 2006; Hassan et al. 2005; Koyasu et al. 2009; Ortiz-Villafuerte \& Hassan 2006) to investigate the influence of void fraction on liquid turbulence parameters. These studies elucidate the phenomenological events important for the modelling of two-phase flows. However, there appears to be a scarcity of subcooled boiling experimental studies that can capture instantaneous whole-field measurements with a fast time response. As an answer to the need of high quality and reliable experimental information of subcooled boiling flow, Estrada-Perez (2004) performed PTV experiments in a square vertical channel with a single heated wall. Their results confirmed the behaviour found in previous studies and provided new information due to the full-field nature of the technique. The new provided information has already been used towards the development of a twophase wall law and for CFD simulation verification and validation Koncar \& Matkovic (2012). When studying the bubble dynamics in subcooled flow boiling, most of the studies rely on visualization techniques. A summary of such works is presented next. Abdelmessih et al. (1972) used high speed photography to investigate the effect of fluid velocity on the growth and collapse of vapour bubbles in slightly subcooled distilled water in an open loop. The measurements included the description of typical parameters describing the lifetime of a bubble: bubble nucleation frequency, growth, and 
collapse. Similarly Ünal (1976) with high speed film photography measured maximum bubble diameters at different subcooling levels and flow rates. Their results fitted previously proposed correlations for the bubbles diameters.Klausner et al. (1993) used high speed digital photography to measure bubble departure diameters. Also, a theoretical analysis was performed to predict that the expected bubbles departure diameter is strongly influenced by the flow velocity and the wall superheat. Bibeau \& Salcudean (1994) performed bubble dynamics measurements using high speed photography. They concluded that bubbles generated near the onset of nucleate boiling conditions slide for longer distances compared to those at different conditions. The maximum bubble diameter and condensation time are shown to be influenced by the location relative to the onset of significant void. Thorncroft et al. (1998) performed a visualization study of vapour bubble growth and departure in vertical upflow and downflow forced convection boiling. They observed significant differences on the boiling bubbles behaviour depending on the liquid flow direction. They observed that the vapour bubble sliding process was an important enhancing mechanism of the heat transferred from the heater wall to the liquid. Chen et al. (2011) studied the effects of channel size on subcooled boiling. They found an increase of both, heat transfer and bubble nucleation frequency when the channel dimensions were reduced. They also observed bubbles merging on the nucleation site due to increases on heat flux. Ahmadi et al. (2012) observed that at high liquid subcooling close to the onset of nucleate boiling conditions, all nucleated bubbles departed for the heater wall and condensed. For lower subcooling levels, bubbles reattached to the wall after lift-off, consequently the bubble lifetime was increased. Chu et al. (2011) measured the bubble lift-off diameters and bubble nucleation frequency in terms of heat flux, mass flux, and degree of subcooling. From this data they suggested a correlation for the bubble nucleation frequency and bubble lift-off diameters. Euh et al. (2010) focused on measurements of bubble departure frequency as a function of pressure, heat flux, flow rate and subcooling level. They developed an automatic image processing technique to obtain the bubble departure frequency. Their measured data was compared with bubble frequency models available in existing literature and an improved version was proposed. Zhou et al. (2013) analyzed the behaviour of single bubbles in a narrow vertical rectangular channel. They showed that at high subcooled conditions nucleated bubbles stick to the wall and slide slowly. From their analysis they suggested that thermophoresis contributes largely to promote the bubble detachment from the wall and that this contribution increases at conditions with high liquid temperature gradients.

\section{Near Wall Measurements and Modeling}

From experimental information researches had explore the relationship between the gas phase parameters with the near-wall liquid velocity. Although the scarcity of detailed experimental information is evident, multiple efforts for the modeling and prediction of two-phase flows have been performed. Here we present a summary of previously proposed models and simulation studies, emphasizing those focusing on the development of a near-wall liquid velocity model. Marie et al. (1997) performed experiments of a turbulent boundary layer developing on a vertical flat plate in the presence of millimeter bubbles. For the bubble frequency and void fraction measurements they used a single-probe optical sensor with a measuring volume of the order of $(50 \mu \mathrm{m})^{3}$, and a high speed camera to measure average bubble velocity, size and shape. For the liquid measurements, they used a miniature conical hot-film anemometer with a sensitive ring size of about $0.1 \mathrm{~mm}$. Also, using a wall flush-mounted hot-film sensor they measured the wall shear stress at a station located at $1 \mathrm{~m}$ from the leading edge. From their detailed measurements, they found that the behaviour of the liquid velocity in the near-wall region consisted on the three regions also found in the single-phase boundary layer theory; the viscous sublayer, logarithmic zone and the wake region were still preserved for their bubbly flows, at least for the void fractions they considered $(\alpha<7 \%)$. They showed that the measured two-phase friction velocity $\left(U_{*}^{\prime}\right)$ cannot be considered as a velocity scale for the description of the level of shear stress in the near-wall region, instead, a new two-phase flow velocity scale was proposed $\left(U_{*}^{\prime x}\right)$ which accounted for the modification of the friction at the surface and the differential gravity forces acting between the wall and the free-stream bubble layers with $U_{*}^{\prime x 2}=U_{*}^{\prime 2}-g\left(\epsilon_{*}-\epsilon_{E}\right) \bar{D}_{B}$ on which $\epsilon_{*}$ and $\epsilon_{E}$ represent the near-wall and the free stream average bubble void fraction values, and $\bar{D}_{B}$ represents the average bubble diameter. Hence, Marie et al. proposed a modified law of the wall 
$U^{+x}=U_{L} / U_{*}^{\prime x}=1 / \kappa^{x} \ln \left(y^{+x}\right)+B^{x}$ with coefficients $\kappa^{x}$ and $B^{x}$ which depend also on the void fraction profile and the average bubble diameter. Relevant of the proposed model is the fact that the slope of the logarithmic law remains unchanged, while its origin is shifted upward depending on the void fraction.Gabillet et al. (2002) performed bubbly flow experiments through a horizontal channel to simulate the effects of wall bubble nucleation and departure through a porous plate in the lower wall. To measure the void fraction, bubble velocity and diameter they used a fibre-optic probe. The liquidphase parameters were measured with a hot film anemometry down to a minimum distance from the wall of about $y=0.75 \mathrm{~mm}\left(y^{+}=45\right)$ due to the probe size restrictions. They showed a self-similar behaviour of the void fraction profiles proportional to the initial void fraction at the injection position $\left(\alpha_{0}\right)$ and the axial distance $(x)$. They also found that the velocity profiles follow a logarithmic law with a behaviour similar to surface roughness studies, suggesting that bubbles acted as roughness elements on the liquid flow. From these observations and from the mixture momentum equation they concluded that a new velocity scale $\left(U_{*}^{\prime} x\right)$ and a new additive constant $\left(B^{x}\right)$ were needed. Their proposed velocity scale being a function of the single-phase friction velocity (measured from pressure measurements), the bubble layer thickness, and the initial void fraction at the injection position $U_{*}^{\prime} x=f\left(U_{*}, \delta^{\prime}, \alpha_{0}\right)$. The new additive constant was defined as a function of the new friction velocity and the indirect measure of the bubble diameter through the bubble induced roughness height $B^{x}=f\left(U_{*}^{\prime}, k_{B}\right)$. With their proposed logarithmic function they obtained similar results as Marie et al., in the sense that the slope of the logarithmic law remained unchanged, however the profile origin shifted downwards instead of upwards. In other modeling efforts,Troshko \& Hassan (2001b) developed a two-phase logarithmic law of the wall for isothermal bubbly turbulent boundary layers. They defined a velocity scale similar to the one used by Marie et al. $\left(U_{*}^{\prime x}=\beta U_{*}^{\prime}\right)$ with a different proportionality factor $(\beta)$ function of the slip and friction velocity, void fraction, and the Von Karman constant $\beta=f\left(U_{r}, U_{*}^{\prime}, \alpha_{\max }, \kappa\right)$. with a slip velocity given by $U_{r}=\left(4 g \sigma \Delta \rho / \rho_{l}\right)^{1 / 4}\left(1-\alpha_{m} a x\right)^{3 / 4}$. An important part of their model was a correction for high void fraction cases on which the linear superposition assumption of the total turbulent viscosity was under question. This correction was included into the $\beta$ proportionality factor with the term $\kappa_{l}=4.9453 \exp \left(-40.661 \cdot U_{*}^{\prime}\right)$ which was empirically obtained from the experimental results of Marie et al. (1997).

Using perturbation theory, Mikielewicz (2003) developed a near wall function for isothermal bubbly flows based on the asymptotic correction methodology. He proposed two approaches, one on which the void fraction distribution was assumed constant and the other with a model to predict the wall peaking effect for the condition evaluated. The assumption of a constant void fraction led to a logarithmic function dependent on gas phase parameters such as the void fraction, bubble diameter, and the bubble drag coefficient, while the dependence on the liquid parameters was introduced by the relative velocity and the friction velocity $U_{+}^{x}=f\left(\alpha, D_{B}, C_{D}, U_{r}, U_{*}^{\prime}\right)$. Worth noting is the use of different values for the additive constant $\left(B^{x}=7.7\right)$, and for the location of the transition between the viscous and the logarithmic layers from $y_{+}^{0}=11$ to a value of $y_{+}^{0}=8$, these values justified from the assumption that at locations bellow $y_{+}^{0}$ no bubbles should exist. Mikielewicz tested his approaches against the experimental data of Marie et al. (1997) and found that while there was not a significant difference between the two proposed approaches, both improved the prediction of the two-phase liquid velocity compared to single-phase models. Based on the idea originally proposed by Gabillet et al. (2002) of considering nucleating bubbles as a surface roughness, Ramstorfer (2007) proposed a model for subcooled flow boiling. Firstly, they performed boiling flow experiments through a horizontal channel of about $36 \times 36 \mathrm{~mm}^{2}$. They used high speed visualization to measure bubble size and dynamics, and a 2D laser-Doppler anemometer for the liquid velocity measurements. Two important differences to (Gabillet et al. 2002) approach consisted on the fact that the additive constant ( $B^{x}=B=5.45$ ) was left unchanged; instead a new additive term was included $\left(\Delta u^{+}\right)$which depended on a bubble equivalent roughness defined as $k_{B}=\eta \bar{D}_{B}\left(q_{n b} / q_{w}\right)^{\xi}$ being $\eta$ and $\xi$ coefficients determined experimentally and $q_{n b} / q_{w}$ representing the ratio of nucleate boiling component to the total heat flux through the heater wall, Ramstorfer (2007) obtained, for each condition, the value of the required velocity scale $\left(U_{*}^{\prime}\right)$ and the value of the bubble equivalent roughness $\left(k_{B}\right)$ from fitting the surface roughness law of the wall to the experimental data. Their 
results show a similar behavior to those on isothermal bubbly flows in a horizontal arrangement with a constant slope and a downward shift proportional to the bubble diameter and void fraction. Aligning their modeling to the roughness theory, they defined a velocity scale for boiling conditions function of the bulk velocity $\left(U_{b}\right)$ and a friction coefficient $(f)$ as $U_{*}^{\prime x}=\sqrt{f / 8} U_{b}$ where $f$ is a modified version of the Colebrook and White's friction universal formula (Colebrook 1939), which is dependent on the bubble equivalent roughness. They found that the proposed model was limited to predict conditions of high bulk velocities on which the bubble buoyancy effects on the liquid velocity can be considered negligibly small. To predict the conditions on which the model is valid, they proposed a non dimensional map using the Froude $(F r)$ and the $(J a)$ Jakob number. They found an empirical expression for the boundary that separates the buoyancy independent from the buoyancy dependent regimes, which reads $\mathrm{Fr}=[1-0.47 \log (\mathrm{Ja})]^{-1}$. A summary of some of the proposed two-phase flow models to describe near-wall velocity is depicted in Table . It is important to note that regardless of the nature of the system, isothermal bubbly flow or boiling flow, the preferred model is of logarithmic nature. The analogy to the single phase case of different regions still holds, with slight variations on the location dividing the viscous sublayer and the logarithmic region, either $y_{+}^{0}=11$ or $y_{+}^{0}=8$, these values being somehow arbitrary. Nevertheless the models seem to provide reasonable improvements when comparing to single phase models. Two approaches have been identified, the first approach is the extension of the single phase mixing length theory to predict two phase flows, and the second approach is an analogy to the roughness theory in turbulent flows through channels. Common to these models is the fact that they are based on experimental measurements on which buoyancy effects are much smaller than inertial forces; therefore, considering slow moving bubbles as equivalent roughness in the wall is justified. Also most of the included physics that describe the bubble behavior are based on a balance of forces that a single bubble is subject to, (drag forces, lift forces, buoyancy forces, etc.), however the experimental works used on the development of these models are limited and these assumptions cannot be explicitly tested. Remarkably, multiple attempts have been made to modify the isothermal two-phase flow models to be used on boiling flow modeling (Koncar \& Krepper 2008; Koncar \& Tiselj 2010; Ramstorfer 2007). From these works Ramstorfer et al. (2008) has been the only researchers who performed boiling experiments to support their proposed model. However, they used boiling bubbles as tracers to acquire their liquid velocity measurements, this under the assumption of a zero slip velocity between the phases. This indeed may produce undesirable velocity bias when the bubbles buoyancy play a significant role, consequently, his model is restricted to conditions of high Reynolds numbers and relatively small void fractions.

Table 1 Summary of proposed near-wall functions for two-phase flow simulations

\begin{tabular}{|c|c|c|c|c|}
\hline Parameter & Marie (1997) & Troshko (2001) & Mikielewics (2003) & Ramstorfer (2008) \\
\hline general form & $U_{+}^{x}=\frac{1}{\kappa^{x}} \ln \left(y_{+}^{x}\right)+B^{x}$ & $U_{+}^{x}=\frac{1}{\kappa^{x}} \ln \left(y_{+}^{x}\right)+B^{x}$ & $U_{+}^{x}=-\frac{1}{\kappa^{x}} \ln \left(\kappa^{x} / y_{+}^{x}+M \alpha \kappa^{4}\right)+B^{x}$ & $U_{+}^{x}=\frac{1}{\kappa^{x}} \ln \left(y_{+}^{x}\right)+B^{x}-\Delta U_{+}^{x}$ \\
\hline velocity scale & $U_{.}^{\prime x}=\beta U_{.}^{\prime}$. & $U_{.}^{\prime x}=\beta U_{.}^{\prime}$. & $U_{.}^{\prime x}=U_{.}^{\prime}$ & $U_{*}^{\prime x}=\sqrt{f / 8} U_{b}$ \\
\hline correction factor & $\beta=\left[1-\left(g \frac{D_{M}}{v_{0}^{\prime 3}}\right)\left(\epsilon_{P}-\epsilon_{E}\right)\right]^{1 / 2}$ & $\beta=\left[\left(1+\frac{\kappa l \alpha_{\max } U_{r}}{\kappa U !}\right)\left(1-\alpha_{\max }\right)\right]^{-1}$ & $\beta=(1-\alpha)^{1 / 2}$ & $f=\left[-2 \log \left(\frac{2.51}{R e \sqrt{ } J}+0.27 C_{k r e} \frac{k_{t}^{+}}{D_{\Lambda}}\right)\right]^{-2}$ \\
\hline additive constant & $B^{x}=B+y_{+}^{0}\left(\frac{1}{\beta}-1\right)-\frac{1}{\kappa} \ln (\beta)$ & $B^{x}=y_{+}^{0}(1-\beta)+\beta B$ & $B^{x}=7.7$ & $B^{x}=B$ \\
\hline Von Karman constant & $\kappa^{x}=\beta^{-1} \kappa$ & $\kappa^{x}=\kappa \beta^{-1}$ & $\kappa^{x}=\beta \kappa$ & $\kappa^{x}=\kappa$ \\
\hline end of viscous layer & $y_{+}^{0} \approx 11$ & $y_{+}^{0} \approx 11$ & $y_{+}^{0} \approx 8$ & $y_{+}^{0} \approx 11$ \\
\hline$U_{+}^{x}=U_{L} / U_{\bullet}^{\prime}$ & $y_{+}^{x}=y U_{*}^{\prime z} / \nu$ & $\kappa_{t}=4.9453 e^{-40.661 U^{\prime}}$ & $M=\frac{3 C_{D} U_{r}}{4} \frac{\nu}{\bar{D}_{B} \kappa^{2}} \frac{U_{*}^{\prime 4}}{4}$ & $\Delta u_{+}^{z}=\frac{1}{\kappa} \ln \left(1+C_{\mathrm{kr}} k_{r}^{+}\right), k_{r}^{+} \geq 90$ \\
\hline$\kappa \approx 0.419$ & $B \approx 5.45$ & $U_{r}=\left[4 g \sigma \Delta \rho / \rho_{l}\right]^{1 / 4}\left(1-\alpha_{\max }\right)^{3 / 4}$ & $C_{D}=\frac{24\left(1+0.15 \mathrm{Re}^{0.687}\right)}{\operatorname{Res}}\left(1+\frac{0.02 D_{B}}{y}\right)$ & $k_{r}^{+}=\eta \bar{D}_{B}\left(\frac{q_{\mathrm{nb}}}{q_{\mathrm{w}}}\right)^{\varepsilon}$ \\
\hline$C_{\mathrm{krc}} \approx 0.65$ & $C_{\mathrm{kr}} \approx 0.65$ & & $U_{r}=\left[\frac{2 \sigma}{\rho_{l} D_{B}}+\frac{\Delta \rho g D_{s}}{2 \rho_{l}}\right]^{1 / 2}$ & \\
\hline
\end{tabular}

Common to all previous proposed models are the efforts to develop a near-wall function based on a logarithmic model assumption. This is indeed a common sense choice, considering how well the law of the wall predicts single phase turbulent flows. However, such assumption has led to new and different constants or empirical correlations among researchers. Some of the inconsistencies among these models are due in part to the lack of detailed experimental information. The flow boiling phenomenon complexity does not easily allow measuring the relationship between the phases in a simultaneous and instantaneous manner. Most of the experimental data on flow boiling are non- 
simultaneous average measurements of either the liquid and/or the gas phases (Lee et al. 2002a; Roy et al. 2002; Yeoh et al. 2002a), making it difficult to find proper relationships between them. This lack of detailed information is part of the motivation of this work.

Although a large number of studies are available in the literature, subcooled flow boiling is still not completely understood. Due to experimental and technical difficulties, most of these studies are often oriented to measure parameters of only one phase, i.e. either liquid or vapour parameters, with experimental approaches that are also limited to offer either long-term averages, such as average void fraction distribution, average velocity profiles, etc. or instantaneous or fast occurring events, such as bubbles departure frequency, bubble growth rate, instantaneous liquid vorticity fields, etc. Furthermore, there had been limited attempts to capture simultaneously the full-field wall heat transfer mechanisms and the interaction with the liquid and vapor dynamics. The present study is a continuation of the experimental endeavor started by Estrada-Perez and Hassan (2010), and has the objective of extending the measurements capabilities to overcome some of the measurements limitations as to include simultaneous PIV, Shadowgraphy and Infrared thermometry to fully account for local and global changes present in subcooled flow boiling. This work is an attempt to provide high-quality data of turbulent subcooled boiling flow for validation and improvement of two phase flow computational models.

\section{EXPERIMENTAL SETUP}

The experimental facility was designed for the visualization of subcooled boiling flow of refrigerant HFE-301 at low system pressure. The facility consists of a hydraulic loop and a visualization system. Experimental setup details are given in the following sections.

\section{Hydraulic Loop}

The hydraulic loop consists of an external loop and a test section, both designed to withstand temperatures in excess of $200{ }^{\circ} \mathrm{C}$, and pressures up to 100 psi. The external loop provides thermal and hydraulic steady state conditions. The system excess energy is removed with a small plate heat exchanger connected to a chilling system. This allows us to have control of the inlet temperature to the test section. The mass flow rate to the test section was measured with a Coriolis flow meter and controlled by adjusting the test section valves. The test section is a rectangular channel made of transparent polycarbonate, with $30.5 \mathrm{~cm}$ length and a cross-sectional area of $10 \times 10 \mathrm{~mm}^{2}$. Energy for boiling is provided through a transparent ITO heater with a length and width of $25.4 \mathrm{~cm}$ and $9.8 \mathrm{~mm}$, respectively, and a maximum working temperature of $200{ }^{\circ} \mathrm{C}$. The heater is attached to the interior face of the channel (see Figure 1 (left)). The electric current to the heater is provided and adjusted by a DC power supply, from which a maximum wall heat flux of $10 \mathrm{~kW} / \mathrm{m}^{2}$ was measured. To reduce heat losses to the ambient, an insulation system made of transparent polycarbonate and Barium fluoride windows covered the test section. The insulation system material was chosen to provide visual access for both the IR-cameras and the High speed camereas. An unheated length of about $61 \mathrm{~cm}$ was considered to ensure fully developed flow. To measure the heater wall temperature, a midwave infrared high-speed camera (3-5 $\mu \mathrm{m})$ was used. A K-type thermocouples is attached to the external face of the heater, to provide validation for the Infrared camera measurements. The test section fluid inlet $\left(T_{\text {in }}\right)$ and outlet ( $T_{\text {out }}$ ) temperatures are measured with two fast response T-Type thermocouples. Figure 1 shows the schematics of the hydraulic system and the dimensions of the test section. 

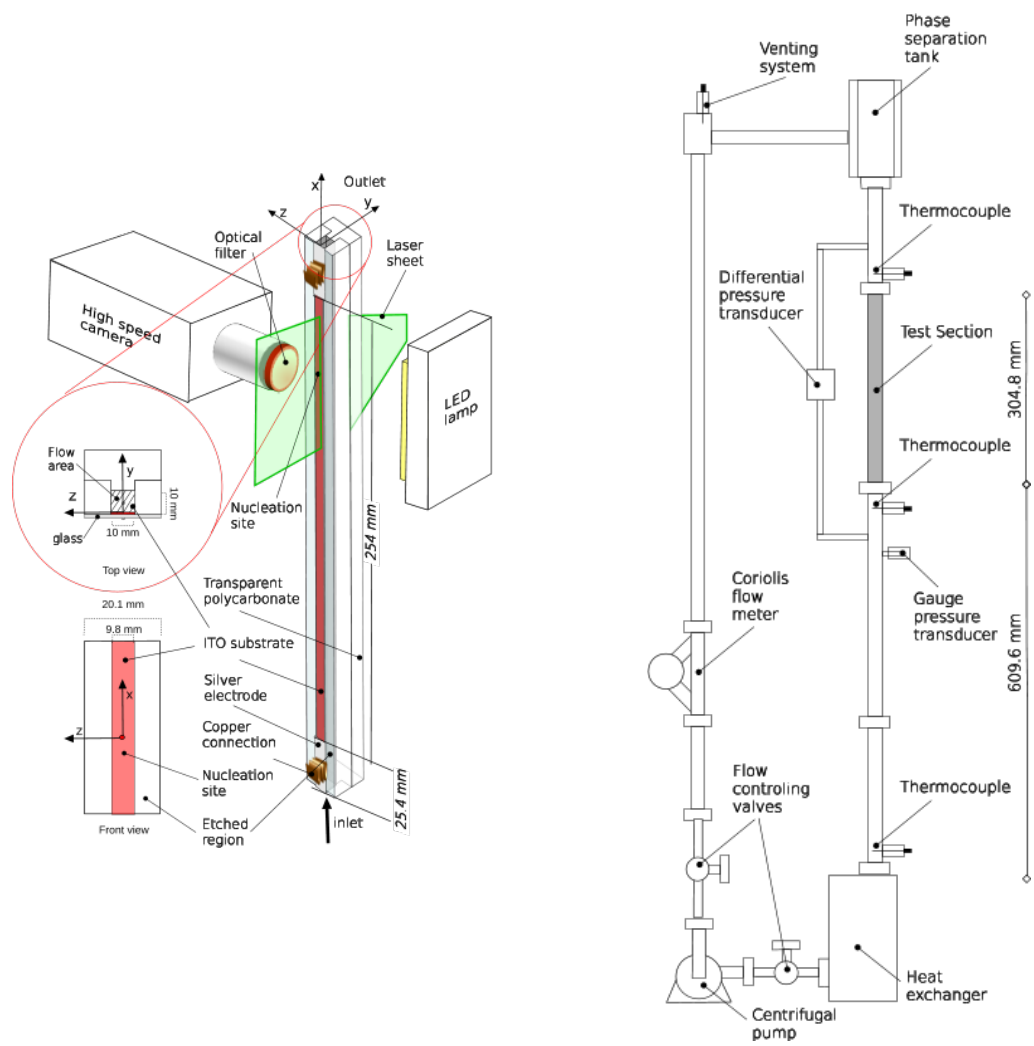

Figure 1 Test section (left), hydraulic loop (right).

\section{Visualization System}

The visualization system consisted of a high-speed high-resolution cameras, a high-speed high-power laser, a high-speed infrared camera, a fast response LED illumination system, mirrors, translational stages, lenses, optical filters and particle flow tracers. The flow tracers are fluorescent particles with density range of 1.39 to $1.41 \mathrm{~g} / \mathrm{cm}$, with an average particle diameter of $5 \mu \mathrm{m}$. The high-speed camera has a maximum frame rate of $6000 \mathrm{fps}$ at a resolution of 1024x1024 pixels, with a maximum bit depth of 12 bits. The illumination was provided by a Pegasus dual lamp laser which can operate at a maximum power of $27 \mathrm{~mJ} /$ pulse. A maximum pulse rate of 20,000 pulse/s can be achieved. Two optical mirrors and two concave-convex lenses are used to convert the small circular beam from the laser, into a thin sheet of light. The laser light sheet is positioned on the measurement region, parallel to the camera focal area. The LED system is externally synchronized to be used as the shadowgraphy illumination. The camera, mirrors and lenses are mounted on translational stages to have the capability of changing the measuring region along the test section. Fig. 2 shows the schematics of the visualization system. The two cameras (Infrared camera and the High-speed camera), the laser and the LED illumination system are synchronized in such a way as to be able to obtain quasi-simultaneous PTV and Shadowgraphy images. 


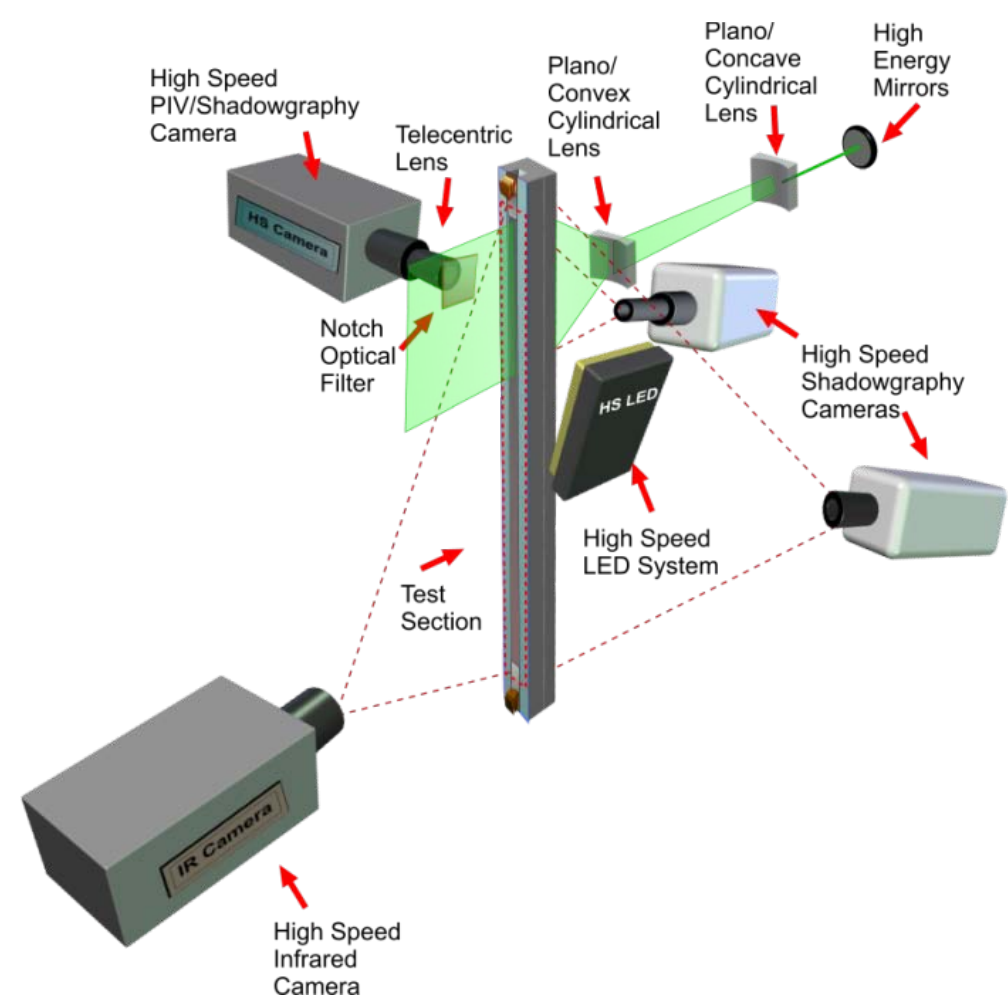

Figure 2 Visualization system schematics

The configuration shown in Figure 2 was designed to have multi-orientation and multi-scale measurements. The high speed PIV-Shadowgraphy camera is arranged to provide "semi-simultaneous" measurements of PTV and shadowgraphy images (see Figure 2). This is achieved by synchronizing the camera shutter with alternating pulses of laser and led illumination. From the temporal multi-scale point of view, this camera can be configured to run in continuous or in straddling modes. Continuous mode provides a fixed time interval between each consecutive frame. In order to properly capture the fast moving features within the flow, this time interval $\left(\Delta t_{\text {PIV }}\right)$ need to be small and consequently the camera need to be run at high speeds. Due to the camera memory limitations the continuous configuration provides short measuring times of the order of fractions of a second. Straddling mode combines a small time interval (small $\Delta \mathrm{t}_{\mathrm{pIV}}$ ) between image pairs plus a waiting period which will be called straddling time $\left(\Delta \mathrm{t}_{\text {straddling }}\right)$. This waiting period is required to acquire a higher number of independent samples while increasing the measuring interval. Similarly, the two high speed shadowgraphy images can also be configured to work in either continuous or straddling modes.

To achieve spatial multi-scale measurements, the PTV-Shadowgraphy camera is used with a telecentric lens which allow the selection of two different visualization modes: High and medium magnification modes, which corresponds to a resolution of 4.82 and $29.1 \mu \mathrm{m}$ respectively. The shadowgraphy cameras are configured to have different visualization modes: one set as high magnification while the other at low magnification. The low magnification corresponds to a resolution of $187 \mu \mathrm{m}$. 


\section{EXPERIMENTS}

\section{PTV-IR-Shadowgraphy experiments}

The measurement area was located at about $23 \mathrm{~cm}$ from the test section inlet. Simultaneous PTV and shadowgraphy measurements were performed at this position with a single high-speed camera. The high-speed camera frame rate was set to 6,000 frames/s, with an exposure time of $150 \mu \mathrm{s}$. The relatively large exposure rate was selected as to allow enough light to reach the camera sensor and achieve satisfactory shadowgraphs of the bubbles. The PIV and shadowgraphy frame rate was 3,000 frames/s. This is due to the selected intermitency in between shadowgraphy and PIV images which allows to take one frame of PIV imediatelly followed by one frame of shadowgraphy. Each acquired image consisted of $1024 \times 1024$ pixels. As of today, only one Reynolds number has been considered $\mathrm{Re}=4200$. For this Reynolds number, about 13 different heat fluxes (q") were imposed ranging from 0.0 to $10.0 \mathrm{~kW} / \mathrm{m}^{2}$. For all cases a constant inlet temperature of $10{ }^{\circ} \mathrm{C}$ was maintained. The heater wall temperature was measured with the infrared camera. The infrared camera magnification allowed temperature measurements of the whole test section. Inlet and outlet fluid temperatures were also measured by means of thermocuples. Figure 3 shows an example of the simultaneous PIVShadowgraphy measurements. Figure 3a shows the image obtained with the laser light ilumination. This is the PIV image which provide information for the liquid velocity measuements. It is important to mention that the camera lens is equiped with a notch filter with a band pass within the range $527+/$ $15 \mathrm{~nm}$. The notch filter blocks most of the laser ligth, and therefore helps to reduce the undesired reflections from the bubbles. This in turns allows a more accurate liquid velocity measurement, since the spurius vectors produced by the bubbles reflections are notabely reduced. Figure $3 \mathrm{~b}$ shows the images acquired with the LED ilumination.
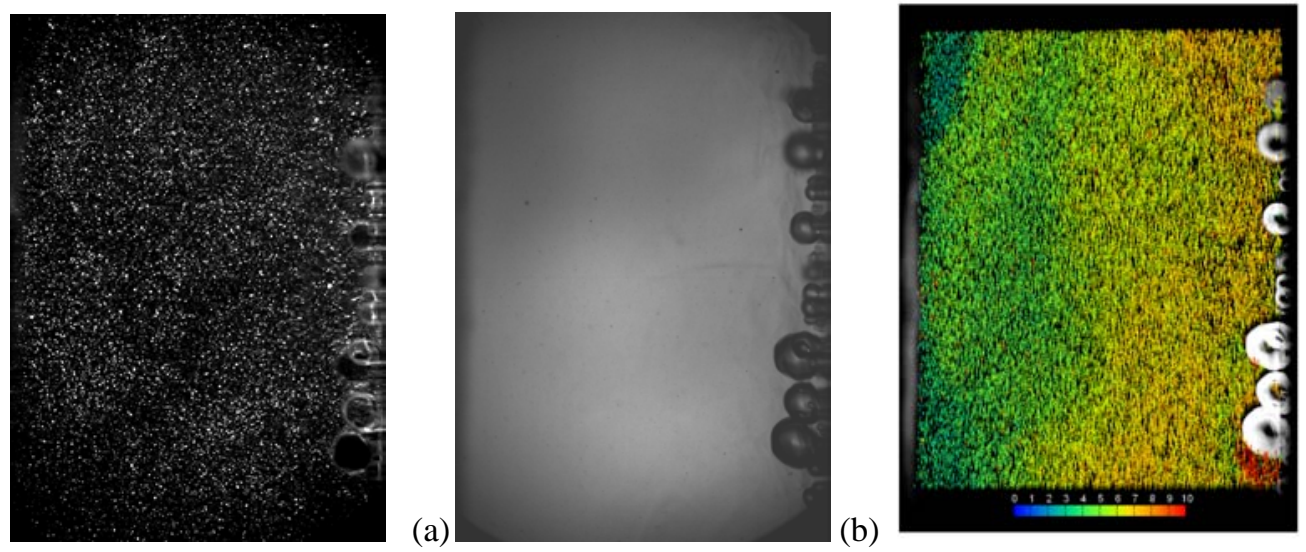

(c)

Figure 3 Experimental results showing a) PIV image, b) Shadowgraphy image c) Velocity field obtained with PTV

These are the shadowgraphy images which will provide the gas phase dynamics. By combining the information from the PIV and shadowgraphy images, a more faithful liquid velocity measurement can be obtained. The bubbles images from the shadowgraphy frames are used to reduce even more the bubbles reflections that are still present in the PIV frame. This will provides a cleaner version of the PIV image on which the bubbles reflections are efficiently removed. The PTV analysis of the "clean" PIV frame is shown in Figure 3c. No velocity vectors were obtained at positions fully occupied by bubbles, confirming that only the liquid velocity is being tracked. It is also noted that the velocity magnitude is larger in regions close to the heated wall (right part of the channel). Figure 4 shows the Infrared thermometry and Shadowgraphy measurements along the whole length of the heater. 


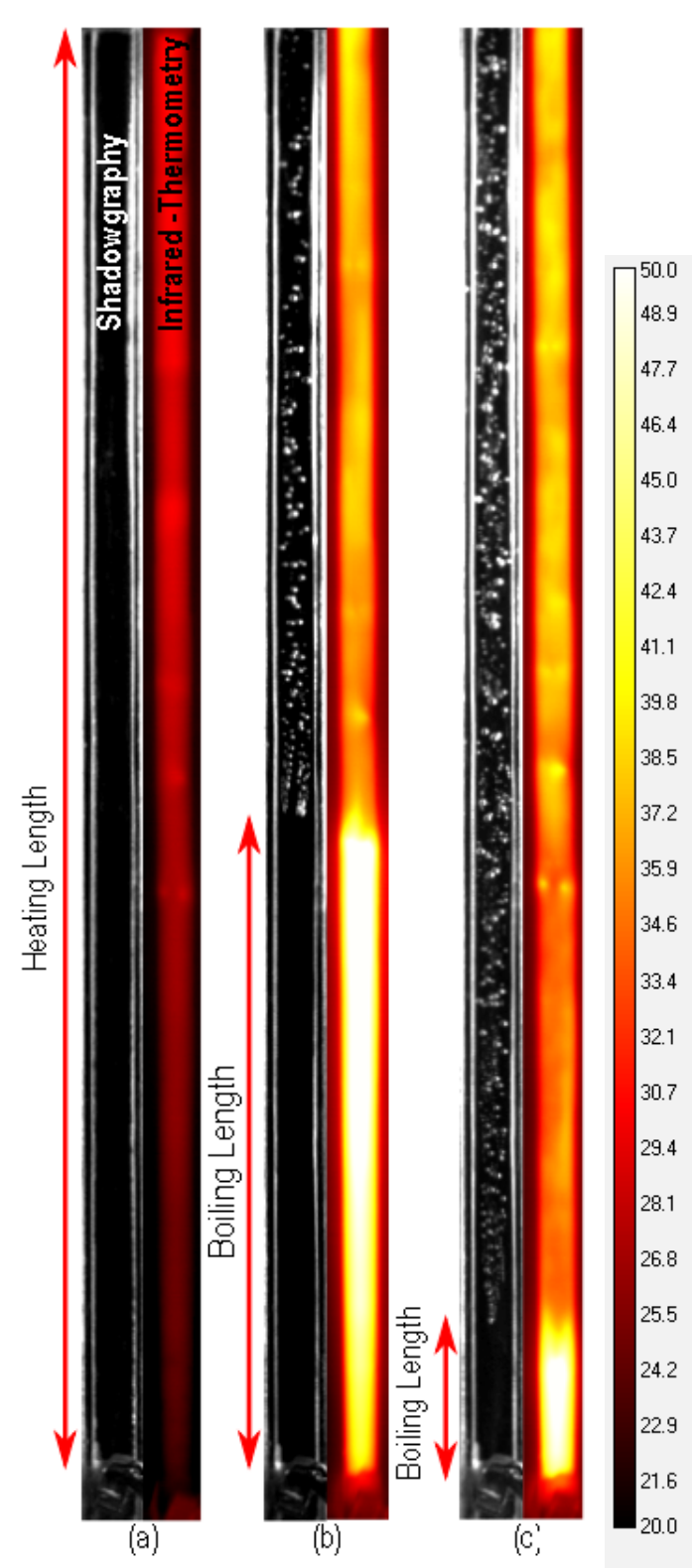

Figure 4 Simultaneous shadowgraphy and infrared thermometry

The shadowgraphy images shows the bubbles as white-brigth spots in a black background. The transparency of the ITO heater allows visual access to achieve these measurements. The infrared images are shown next to the shadowgraphy images for a better understanding of the effect that nucleation sites location have on the heater wall temeprature. Figure 4a shows a heat flux condition of $6 \mathrm{~kW} / \mathrm{m}^{2}$ at this heat flux no boiling was present. No large temperature gradients are found. Also it is clear the appearance of slight hot spots which are more evident at the higher heat fluxes cases. Figure $4 \mathrm{~b}$ shows a heat flux condtition of $8 \mathrm{~kW} / \mathrm{m}^{2}$. The onset of nucleate boiling at the middle of the test section produces a sudden temperature decrease just after the location of the two nucleation sites. The "Boiling Length" which represent the single phase flow portion along the heater, presents a larger temperature than the observed after the "Boiling Length" section. The large heat transfer induced by the nucleating and sliding bubbles is evident from Figure $4 \mathrm{~b}$ and c. Boiling brough significan changes to the heater wall temperature. The amount of heat removed by the bubbles prescence translates in a 
decrease in temperature. The Infrared camera is shown to have enough sensitivity to detect the temperature variations originated from the bubbles. Further analysis of the IR-Thermometry images is needed to validate the temperature measurements and to perform sound estimations of local heat transfer. Figure 5 shows the liquid flow statistics as obtained from the analysis of the PIV images using the PTV algorithm. Figure 5a shows the axial liquid velocity profile for different heat fluxes. Wall heating brought significant changes in the velocity distribution profiles. Some of these changes are general trends observed also in other studies [12, 30, 14, and 26] and are summarized here: First, the mean liquid axial velocity in regions close to the heated wall increases accompanied with a decrease in the axial velocity for regions far from the heated wall. Second, there is a marked shift of the maximum liquid axial velocity location toward the wall. Figure 5a shows the profiles of mean liquid axial velocity $\bar{U}$ for $\operatorname{Re}=4,200$ with wall heat fluxes ranging from 0 to $10 \mathrm{~kW} / \mathrm{m}^{2}$. Figure $5 \mathrm{~b}$ shows the axial rms profile $\left(u^{\prime}\right)$ for various values of wall heating for $R e=4200$. The heat flux influence is large, including the low heat flux cases. Two trends are observed: first, from low values of $q^{\prime \prime}=5$ to 6 $\mathrm{kW} / \mathrm{m}^{2}$ there is a decrease in the axial rms profiles with respect to the isothermal case, the second trend is observed for the higher heat fluxes where an increase of wall heat flux significantly increase the rms values. Figure $5 c$ show the normal RMS profiles $\left(v^{\prime}\right)$ at various values of wall heat flux for $\operatorname{Re}=4200$. This figure shows similar trends as those found for the axial RMS profiles $\left(u^{\prime}\right)$. Two trends were also observed in Figure 5c. The first heat flux cases showed a decrease on the profile compared to the isothermal case, while the rest of the heat flux cases presented a large increase on RMS due to the wall heating. The Reynolds stresses $\left(u^{\prime} v^{\prime}\right)$ profiles are shown in Figure $5 \mathrm{~d}$ for Re $=$ 4200. A profile decrease tendency was found as a result of a wall heat flux increment, and a marked shift of the zero location toward the heated wall can be observed. At the beginning of heating a peak reduction and shift of the zero Reynolds stress locations towards the wall was found. Further increase of the heat flux resulted in more reduction in the profile and an inverted peak is observed. The location of this inverted peak (minimum value) will shift towards the center of the channel as a result of the increase of the wall heat flux.

(a)

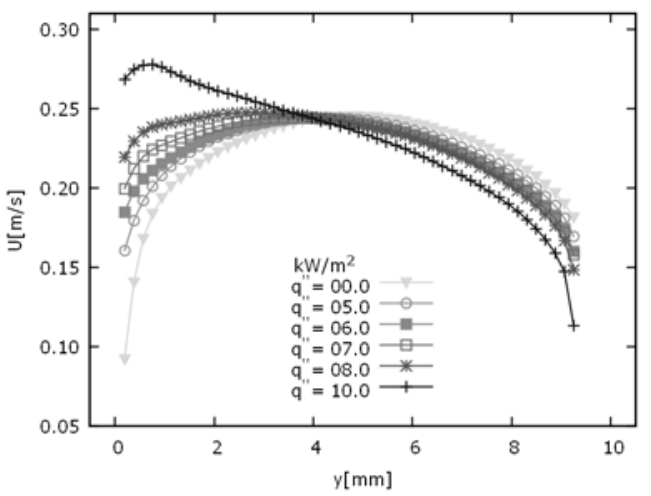

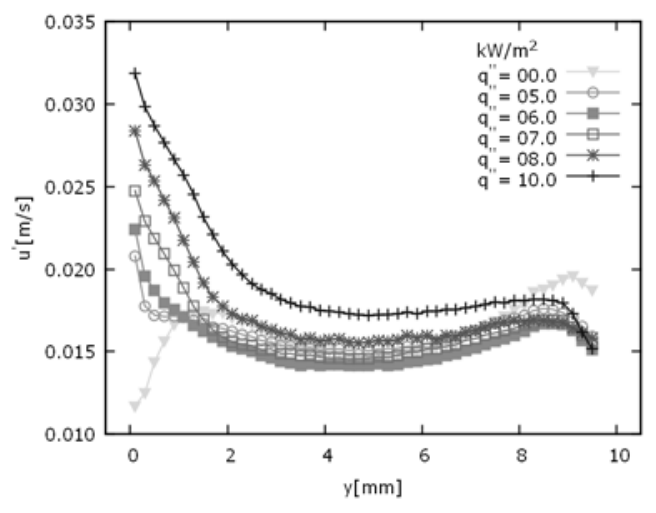



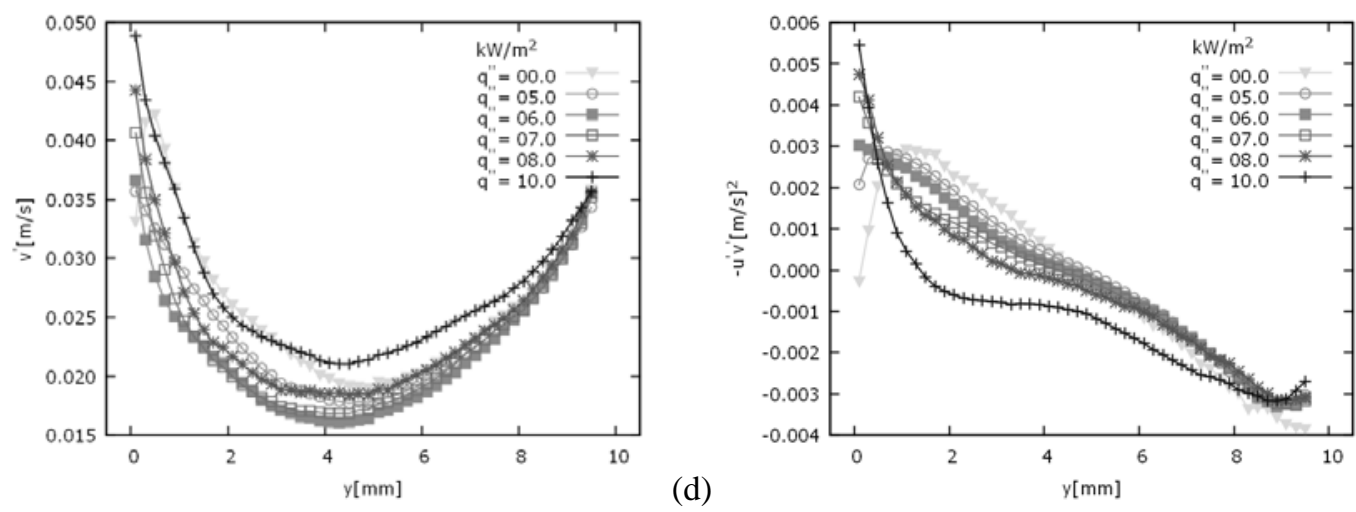

Figure 5. Liquid flow statistics a) Average axial velocity, b) rms of the axial velocity component, c) rms of the normal velocity component, d) Reynolds stresses.

\section{Single Nucleation Site Approach}

These experiments were performed by placing the measurement area on the vicinity of a single nucleation site. In this manner, it was possible to measure the flow development from a single-phase heated condition on its transition to a two-phase bubbly flow condition, i.e., measurements were available for both upstream and downstream of the nucleation site covering the full bubbles life-cycle. After the nucleation site of interest was located, the laser sheet light was positioned to be exactly on top of the nucleation site. In this manner, a snapshot of the instantaneous influence of bubbles parameters on the liquid turbulence can be achieved. Schematics of the PTV laser placement on top of the nucleation site are shown in Figure 2.

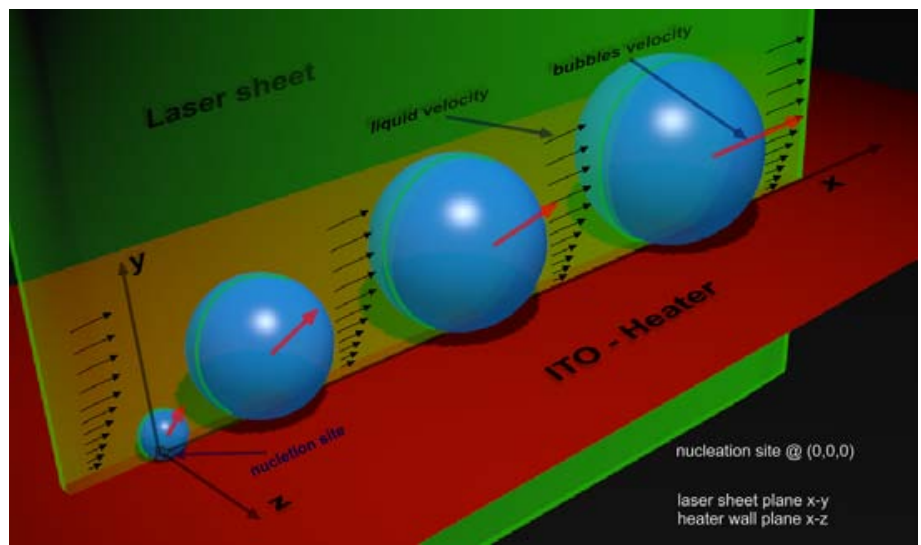

Figure 6 Schematics of PTV laser light location to achieve semi-simultaneous PTV and shadowgraphy measurements.

\section{Shadowgraphy Results.}

Figure 6 shows the results obtained from the high magnification PIV/Shadowgraphy camera. From these results four axial direction profiles of important bubbles parameters are shown. These allows for detailed examination of the average bubble behavior along the bubble path. Four profiles are shown in Figure 6: bubbles width, height, axial velocity, and normal velocity. It is important to note that more than 20000 bubbles are used to show the most probables behavioral bubble paths along the heater wall. Furthermore, these results were obtained with the straddling timing mode, which provides an independent measuring sample for each pair of images. Insight into the benefits of performing multiscale measurements can be gained when comparing the results from Figure 6 and Figure 7 which are 
syncronized measurements at the same conditions but from different view points and visualization modes. Figure 6 shows the high-magnification side-view results, while in Figure 7 are shown the lowmagnification front-view results. Figure 6 provides detailed bubble dynamics information, however this measurement is limited to only $3.5 \mathrm{~mm}$ from the nucleation site. Figure 7 shows less detailed information in a larger scale. To make the differences of scales between these measurements clearer, a small rectangle close to the nucleation site in Figure 7 depicts the measurement region from the sideview high-magnification camera.
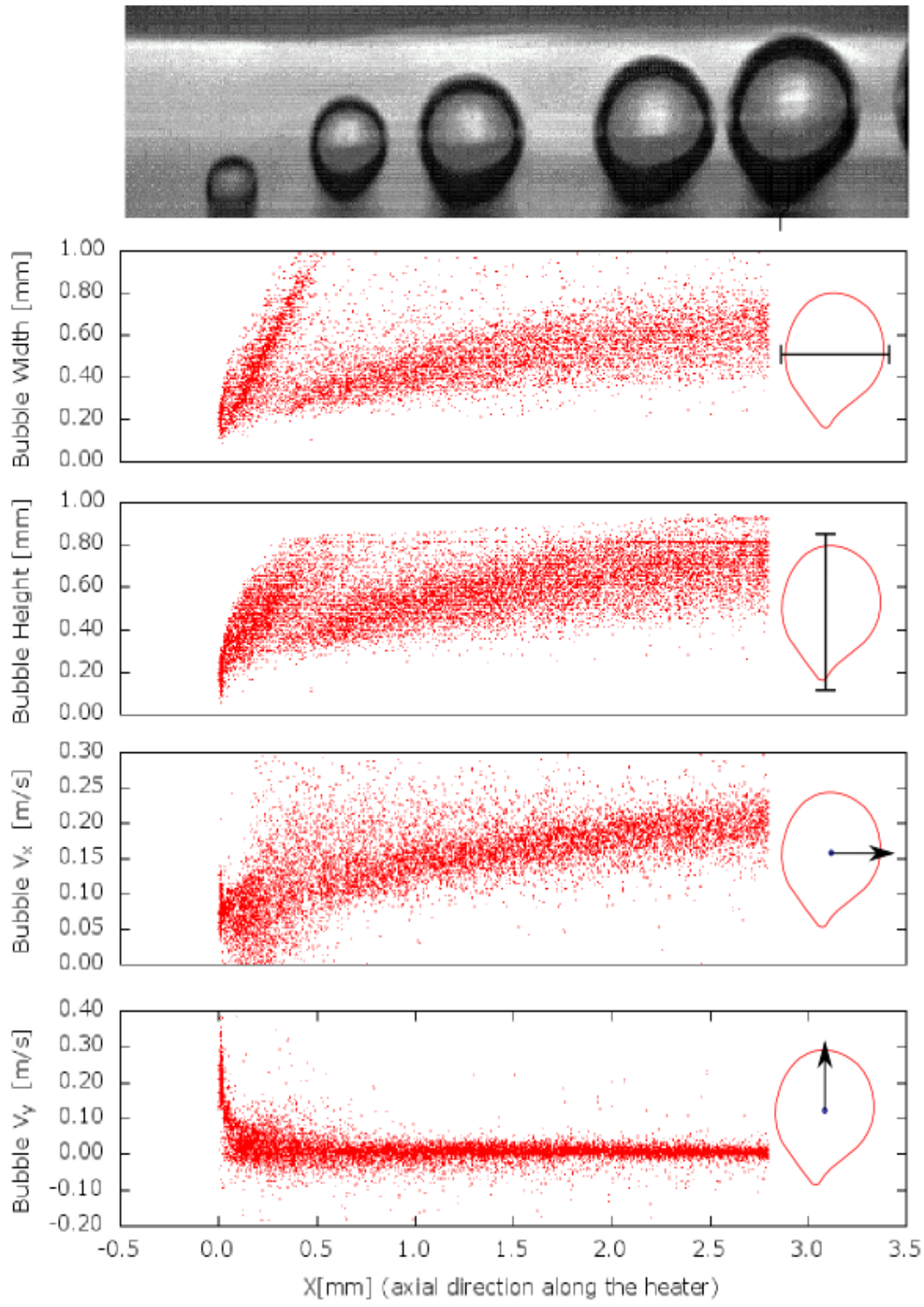

Figure 7 Side view, high magnification bubble dynamics measurements 


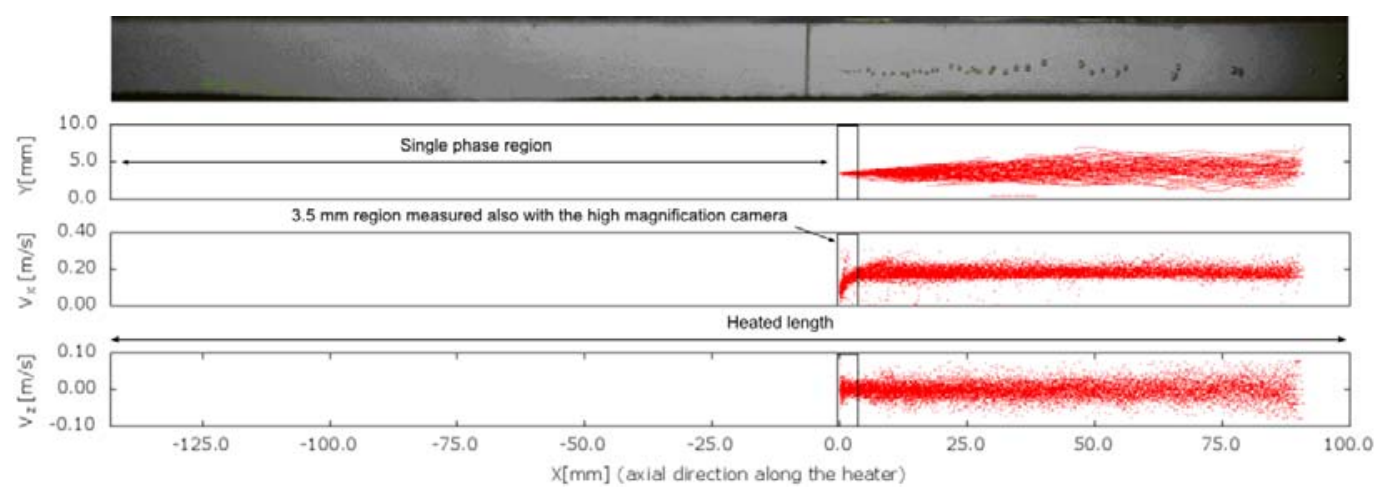

Figure 8 Low magnification shadowgraphy visualization for bubble dynamics measurements.

\section{PTV Results}

Figure 8 and Figure 9 show the particle tracking velocimetry results from the side-view mediummagnification configuration. This configuration was selected to obtain information of the liquid behavior on its transition from before to after the nucleation site. The first plot in Figure 8 shows an instantaneous velocity field on top of the corresponding PIV image. It is important to recall that for these experiments, semi-simultaneous PIV and shadowgraphy measurements were performed. The shadowgraphy results helped to reduce the spurious vector detection on the PIV analysis. This was possible by removing bubbles images and keeping only the liquid information. The vector color in the first plot of Figure 8 represents the 2D velocity magnitude. Using the color criteria, it is clear that before the nucleation site, single-phase fully developed turbulent flow behavior is observed. The second plot of Figure 8 shows the average velocity profiles along the heated wall. There is no significant change on the axial velocity for positions upstream the nucleation site, however, downstream the nucleation site significant changes on the liquid velocities are observed. There is a concomitant axial velocity increase with the increase of the distance downstream the nucleation site. This velocity increase is clearly driven by the momentum induced by the bubbles whose size become more prominent as they go sliding through the wall. Similarly, the third plot of Figure 8 shows the normal velocity profiles along the heater length before and after the nucleation site. The liquid normal velocity seems to be relatively unaffected upstream the nucleation site. However, on the nucleation site vicinity, the normal velocity shows a large increase on regions close to the wall. Bubbles when nucleated, transfer a significant amount of momentum to the liquid. This abrupt increase on the normal velocity is reduced downstream the nucleation site because of the reduced bubble growth rate along the heated wall. Figure 9 shows both the axial and normal RMS profiles next to an instantaneous velocity field for comparisons. The second plot of Figure 9 shows the behavior of the axial velocity RMS ( $u$ ') profiles along the heater. Upstream the nucleation site, in the single phase region, the reduced axial fluctuations are indication of a laminarization due to the wall heating. This phenomena has been observed previously in other studies, and is attributed to the density reduction within the thermal boundary layer (C. E. Estrada-Perez \& Hassan 2010; Wardana et al. 1999; Wardana et al. 1995; Wardana et al. 1994; Wardana et al. 1992). From the nucleation site region and downstream, a concomitant increase of the axial liquid fluctuation is observed for profiles points close to the wall. This was observed also on previous subcooled boiling flow studies (C. E. Estrada-Perez \& Hassan 2010; Končar et al. 2004; Lee et al. 2002b; Roy et al. 1993; Roy et al. 2002; Roy et al. 1997; Yeoh et al. 2002b). A similar behavior is observed on the third plot of Figure 9 for the normal liquid fluctuations. This increase on the turbulence fluctuations is attributed to the continuous stirring and mixing of the liquid induced during the nucleation, growing and sliding of the bubbles. It is important to mention that the single nucleation site experiment provides, in a single experimental condition, both type of flows, namely: single and two-phase flow. Contrary to previous works which focused on fully developed bubble layer regions, on which the measurement area were covered either single or twophase flow. This unique characteristic of measuring the liquid behavior in the transition from a single 
to a two-phase flow condition may simplify the understanding of the impact of important boiling bubbles parameters on the liquid turbulence.

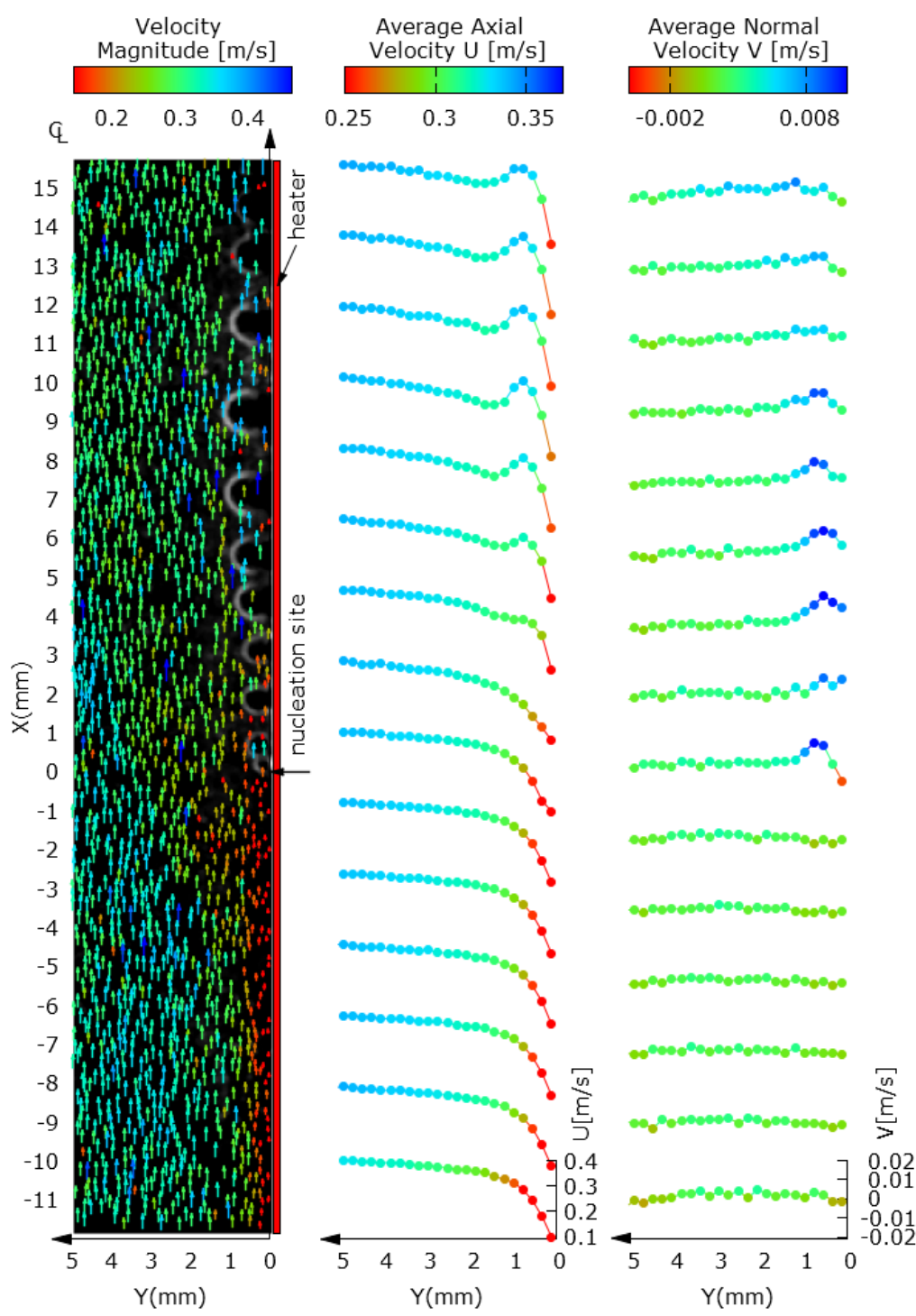

Figure 9 From left to right: Instantaneous velocity field, average axial velocity profiles, and average normal velocity profiles along the heater length. 


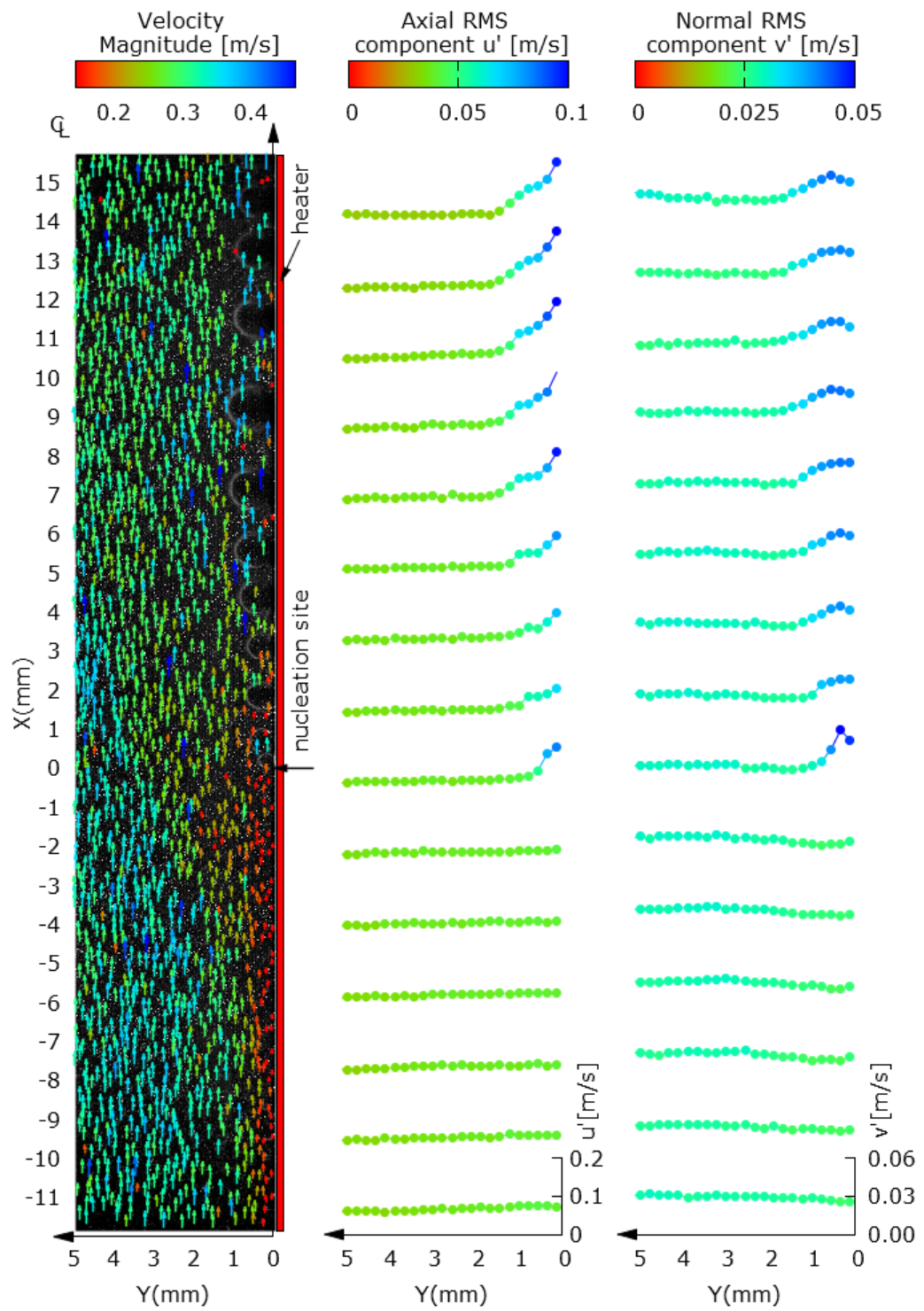

Figure 10 From left to right: Instantaneous velocity field, average axial RMS profiles, and average normal RMS profiles along the heater length. 


\section{Axial liquid velocity modification}

One of the main objectives of the PTV measurements is to obtain detailed information of the liquid velocity close to the heated wall under subcooled boiling conditions. Figure 11 shows the average axial velocity profile at different $x$ axial locations along the heater. The color bar indicate the axial distance from the nucleation site. It is important to mention that the nucleation site location is the reference value for all the following plots, i.e. the nucleation site is located at $x=0, y=0, z=0$. The influence of the boiling bubbles on the liquid velocity is clear. There is a local peak in the region close to the wall. This local peak is related to the average bubble radius measured at each axial location. Closer examination of the average bubble residence time gives a clear indication that the bubble layer do not extend further than $1 \mathrm{~mm}$ from the wall. This position coincides with the limit on which the liquid velocity appear to be influenced by the bubbles induced momentum.

To have a better understanding of the liquid behavior, it is always convenient to present these results in dimensionless form. The characteristic length and velocity used for the non-dimensionalization are $y^{+}=\left(y u_{\tau}\right) / v$ for the characteristic length and $u_{\tau}$ for the characteristic velocity, $u_{\tau}$ is the friction velocity defined as $u_{\tau}=\sqrt{\left(\tau_{w} / \rho\right)}$. The wall shear stress $\left(\tau_{w}\right)$ is obtained experimentally using the approximation for regions close to the wall as $\tau_{w}=\mu d u /\left.d y\right|_{y=0}$. From the previous definitions we found a single phase frictional velocity of $u_{\tau}=0.0125 \mathrm{~m} / \mathrm{s}$. Using the single phase frictional velocity Figure 11 shows the non-dimensional form of the axial velocity profile at different axial positions. From this plot it is clear a significant difference from the single unheated case (shown in red). These differences are attributed to the gravity driven flow induced in the vicinity of the heated wall. The effect of the boiling level in the liquid velocity was previously observed (C. Estrada-Perez \& Hassan 2010). However, the present experiments focused in a single nucleation site, allowing to have different void fractions depending on the axial position $(x)$. Therefore, a single heat flux condition will provide different local void fractions, which simplified greatly the experimental and analysis work. Also, it is clear that the largest influence of the boiling bubbles is confined bellow the buffer layer $\left(y^{+}<30\right)$, affecting significantly the viscous sublayer. The latter observation may be explained from the analysis of the bubbles dynamics. In the viscous sublayer region a reduced amount of void was found. This region is affected mostly by either small bubbles or by the bubbles neck which slide through the wall. The liquid in the viscous sublayer experience an induced momentum due to the slower moving bubbles neck, and at the same time, a deceleration due to the wall induced drag. Liquid in the buffer layer is less affected by the wall drag and experience an increase of momentum by the bubbles. It is interesting to point out that the concomitant velocity increase with distance from the nucleation site $(x)$ is similar to the behavior found previously by C. Estrada-Perez \& Hassan (2010). However, in the present study the profiles variations are shown at different axial distances, instead, the profiles variation found by C. Estrada-Perez \& Hassan (2010) were due to an increase on heat flux. No matter what is the source of profile variation, either $x$ or $q^{\prime \prime}$, in both cases the velocity profile variation seems to a fairly smooth process, with the exception for regions close to the nucleation site. An abrupt change on the velocity profiles was found in the transition from single-phase flow to a two-phase flow condition, i.e. The regions before the nucleation site show an similar velocity profile, and just after the nucleation site region, the liquid velocity profiles are changed abruptly, and transition towards an almost fully developed region far from the nucleation site was achieved downstream. 


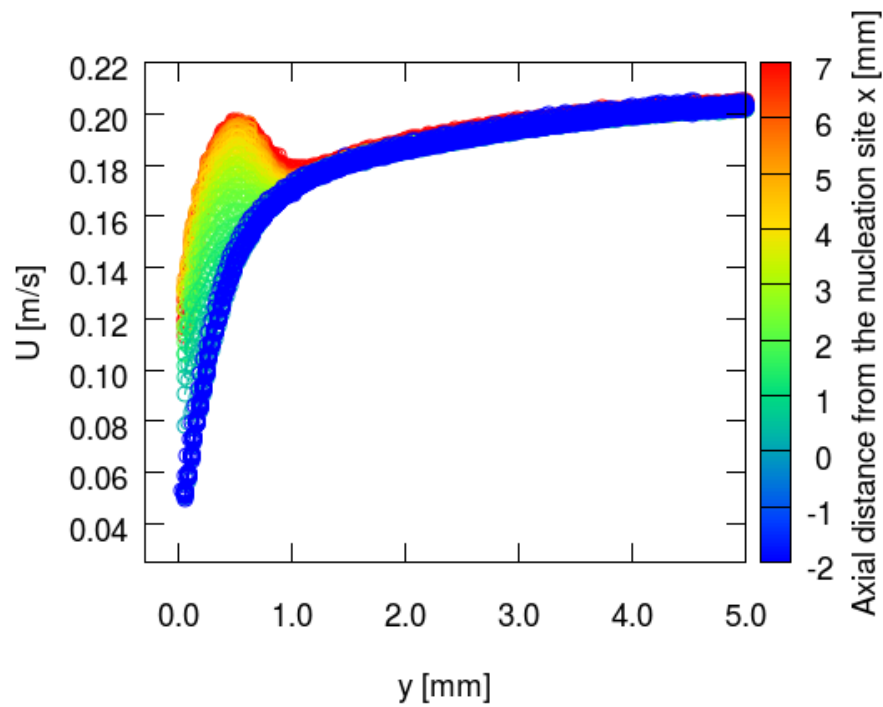

Figure 11 Axial liquid velocity profile at different axial positions from the nucleation site.

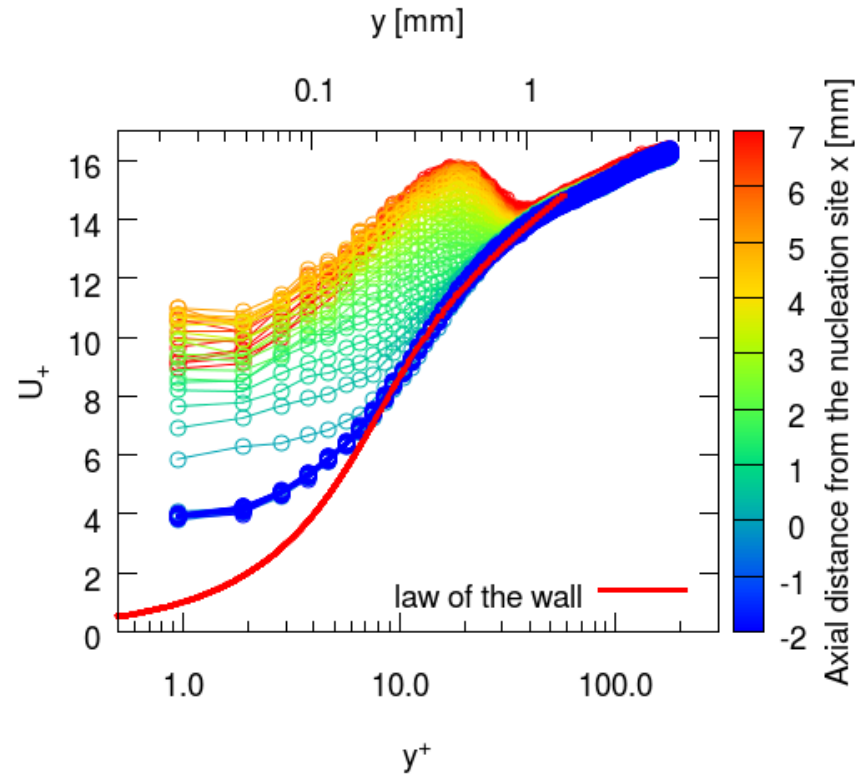

Figure 12 Semi-log plot of the axial liquid velocity profile at different axial positions from the nucleation site using the single-phase friction velocity as a velocity scale.

To further gain insight on the effect of the bubbles parameters on the liquid turbulence, the two phase friction velocity $\left(u_{*}^{x}\right)$ was estimated by three different approaches, namely: The wall-slope method, the Durst polynomial fitting, and from direct differential pressure measurements. These techniques are 
well documented and validated for single phase conditions (Durst et al. 1996), extending these approaches to be implemented in a two-phase condition remains a question. However, these techniques has been previously tested in two-phase flow conditions with some success (Elvis E DominguezOntiveros et al. 2006) . Friction velocity measurements for the single nucleation site experiment were performed using these approaches, Figure 11 shows these results. The wall-slope method and the Durst polynomial approach rely on the accurate estimation of the near wall velocity measurements by the PTV algorithm. Although the reliability of these measurements is reduced when the void fraction increases, the single nucleation site approach allowed us to have enough samples of the near-wall liquid velocity underneath of the bubble layer to ensure sufficient statistics. The Durst method showed to be less sensitive to the selected points to perform the curve fitting, as long as the velocity information was bellow $y_{+}<12$. It is important to note that although significant discrepancies between the Durst and the wall-slope method were found, these measurements were within the differential pressure measurement uncertainty range, giving us some degree of confidence on these results. Also it is important to recall that the wall-slope and Durst methods provide a detailed description of the wall shear stress local change along the heater wall. In this scenario, the differential pressure measurements provide only the average wall shear stress, which is useful for the local wall shear stress measurements validation.

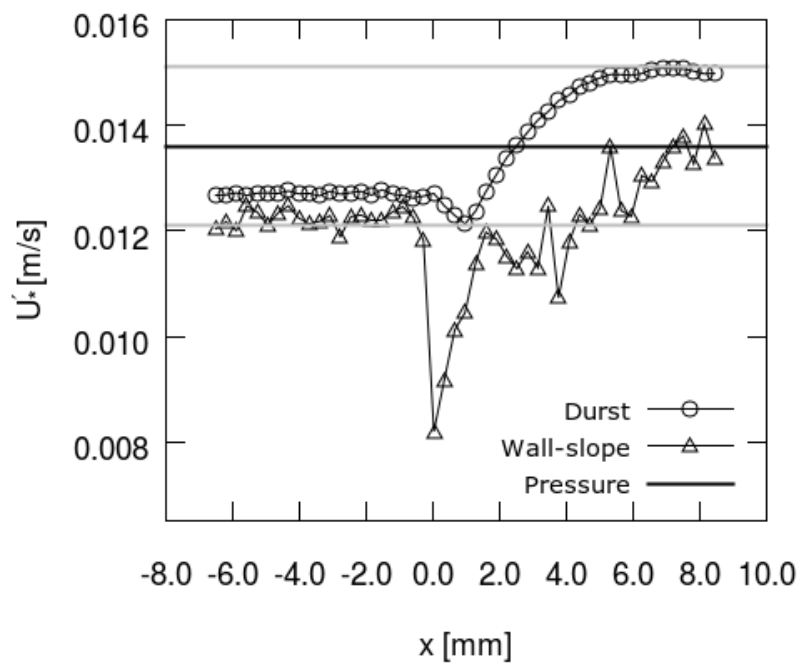

Figure 13 Friction velocity measurements by means of the wall-slope method, Durst polynomial fitting, and from differential pressure measurements.

By using the measured two-phase flow friction velocity the new non-dimensional plot of the liquid velocity profiles are shown in Figure 12. The resulting trend is similar to what Marie et al. (1997) found when using two-phase friction velocity measurements from a wall shear stress sensor. There appears to be a profile downward shifting with an increase of the void fraction. This suggested that the direct use of the measured two-phase friction velocity measurements is not a proper approach to fully characterize the subcooled boiling data. Furthermore, previous studies ((Koncar \& Tiselj 2010; Marie et al. 1997; Troshko \& Hassan 2001a) had focused on the logarithmic layer modeling, i.e. for $y_{+}>30$ and neglecting the viscous sublayer due probably to the lack of experimental data in the near-wall 
region. The simplifying assuming was that a similar dynamics structure prevails for both the single and the two-phase flow conditions. However, when looking to more detailed experimental information it is clear that the viscous sublayer is strongly affected by the bubbles presence, and that this changes cannot longer be ignored.

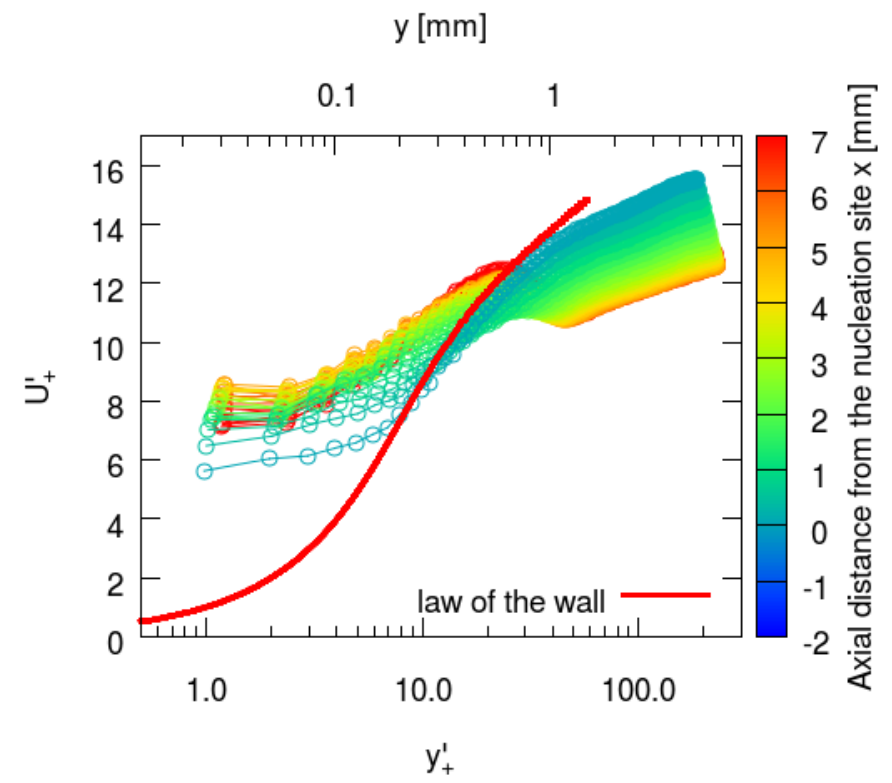

Figure 14 Semi-log plot of the axial liquid velocity profile at different axial positions from the nucleation site using the two-phase friction velocity as a velocity scale.

The availability of the simultaneous and detailed information from both phases allows us to perform parametric studies to test the influence of each gas phase parameters on the liquid turbulence. As an example, an empirical equation was selected to describe the behaviour of the liquid within the bubble layer. This region is governed by two competing factors, the viscous forces and the bubble bouyancy. The first approach was to identify a suitable equation to represent accurately the behaviour within the bubble layer $y<B_{r}$. In this region, most of the bubbles are sliding along the wall surface. During the sliding process, bubbles induced many changes to the liquid turbulence, moreover, the bubbles wake washes away the near-wall liquid, overcoming the viscous forces that keep the liquid from accelerating. These observations lead to the conclusion that, up to the measuring technique resolution, the wall no-slip boundary condition is no longer a proper expression to describe the liquid velocity in this region. In other words, in subcooled boiling conditions, the liquid velocity did not show to approximate a zero value, rather, the liquid moved with a velocity resulting from the bubble sliding,. Empirically, several expressions were fitted to the experimental data using a least square algorithm. This as to provide values for the coefficients which may lead to the understanding of the underlying physics. The first expression tested was $U(y)=A+B y^{(1 / 2)}$. This equation was selected among several multiple options and provided a good fitting for several conditions. Based on the success of this equation, it was decided to include the bubble radius $\left(B_{r}\right)$ as a parameter in this expression. Hence the final expression is as follows $U(y)=B_{r}\left[A+B y^{(1 / 2)}\right]$. By fitting this expression, which included the different bubble radius, it was found that the coefficients $A$ and $B$ are equal with a value $A=B=1 / \kappa_{B}$ with $\kappa_{B}=4$. Then, the near wall liquid velocity can be expressed with

$$
U(y)=\frac{B_{r}}{\kappa_{B}}\left[1+y^{(1 / 2)}\right]
$$


This simple expression appears to work fairly good for all considered cases. However it seems that for the smaller bubbles in the vicinity of the nucleation site this expression under-predicts the data. The behavior of smaller bubbles appears to have little or no contribution due to their low buoyancy. The value of $\kappa_{B}$ is assumed to be a function of the Reynolds number, heat flux, and local void fraction.

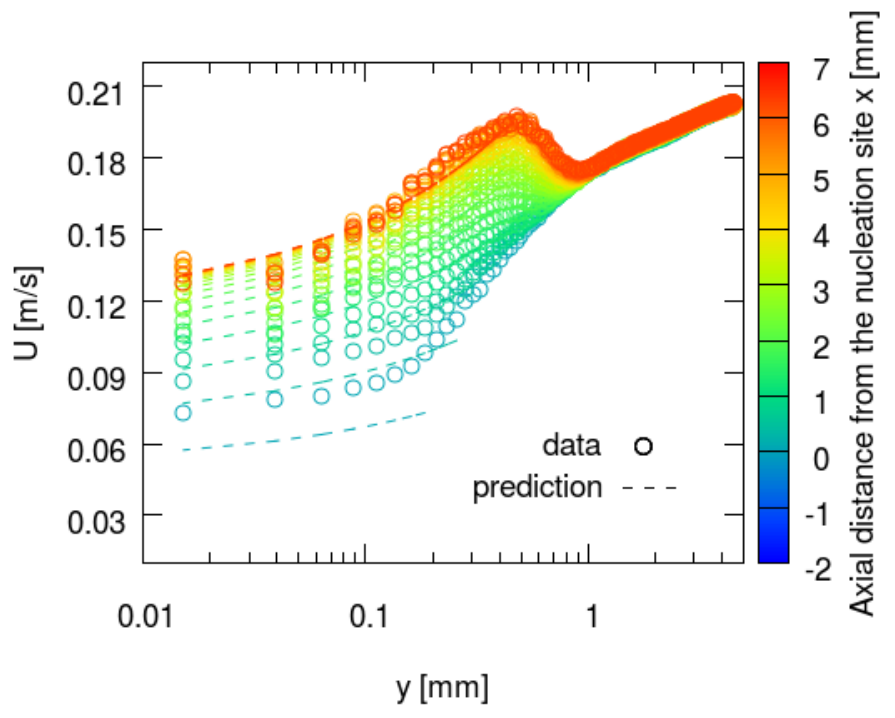

Figure 15 Comparison of velocity profiles and equation (1).

This parametric study although inconclusive is intended as an example of the advantages of these innovative measurement techniques. The availability of the simultaneous measurement is intended to simplify the validation and development mechanistic models for the better description of subcooled boiling flows.

\section{CONCLUSIONS}

In this work is presented the feasible implementation of whole-field visualization techniques such as Infrared Thermometry, Particle Tracking Velocimetry, and High speed Shadowgraphy to study subcooled flow boiling through a vertical square channel with a single heated wall. It was found that although each independent technique requires very specific technical demands, with careful experimental system arrangements, the simultaneous implementation of these techniques to study subcooled flow boiling is viable with reasonable uncertainties and high repeatability. From the PTV perspective several findings/recommendations are highlighted: For typical PTV boiling experiments, the use of high energy lasers in a continuous timing mode has to be used with caution. Laser induced perturbations changed what was considered a stable boiling condition. Therefore, the use of the straddling timing mode is recommended. The straddling timing mode showed to be superior not only due to the reduced laser induced perturbations, but also provided higher quality flow statistics. The straddling timing allowed more independent samples to be used on the estimation of the time average quantities. It was also found that large uncertainties on the PTV measurements were induced by the induced reflections of the boiling bubbles, therefore it is recommended to perform simultaneous PTV/Shadowgraphy experiments. The shadowgraphy experiments will dictate the bubbles domain, from which spurious velocity vectors can be removed to reduce this uncertainties. The experimental methodology developed in this work, provided whole-field velocity fields of the two phases, bubbles dynamics quantification and heater wall temperature fields in a simultaneous manner with acceptable 
accuracy and repeatability. The data gathered using this methodology can be used as an experimental benchmark for validation of CFD codes and for the improvement and development of models and correlations for a better description of subcooled boiling flow.

\section{ACKNOWLEDGEMENTS}

The author acknowledges the contribution of this review paper, present at the conference Computational Fluid Dynamics in Nuclear Reactor Safety (CFD4NRS-5). The contributions of Mr. Carlos Estrada-Perez and Mr. Junsoo Yoo are greatly appreciated. This work is partially supported by the Consortium for Advanced Simulation of Light Water Reactors (CASL) which is funded by the U.S. Department of Energy.

\section{REFERENCES}

Abdelmessih, A., Hooper, F. \& Nangia, S., 1972. Flow effects on bubble growth and collapse in surface boiling. International Journal of Heat and Mass Transfer, 15(1), pp.115-125.

Ahmadi, R., Ueno, T. \& Okawa, T., 2012. Experimental identification of the phenomenon triggering the net vapor generation in upward subcooled flow boiling of water at low pressure. International Journal of Heat and Mass Transfer, 55, pp.6067-6076.

Bibeau, E. \& Salcudean, M., 1994. A study of bubble ebullition in forced-convective subcooled nucleate boiling at low pressure. International journal of heat and mass transfer, 37(15), pp.2245-2259.

Chen, C. et al., 2011. Saturated flow boiling heat transfer of R-410A and associated bubble characteristics in a narrow annular duct. International Journal of Heat and Mass Transfer, 54(23), pp.4988-5000.

Chu, I.-C., No, H.C. \& Song, C.-H., 2011. Bubble Lift-off Diameter and Nucleation Frequency in Vertical Subcooled Boiling Flow. Journal of nuclear science and technology, 48(6), pp.936949.

Colebrook, C.F., 1939. Turbulent Flow in Pipes, with particular reference to the Transition Region between the Smooth and Rough Pipe Laws. Journal of the ICE, 11(4), pp.133-156.

Dominguez-Ontiveros, E.E. et al., 2006. Development of a wall shear stress integral measurement and analysis system for two-phase flow boundary layers. Rev. of Scientific Inst., 77, p.105103.

Dominguez-Ontiveros, E.E. et al., 2006. Development of a wall shear stress integral measurement and analysis system for two-phase flow boundary layers. Review of scientific instruments, 77(10), p.105103.

Durst, F. et al., 1996. Wall shear stress determination from near-wall mean velocity data in turbulent pipe and channel flows. Experiments in Fluids, 20(6), pp.417-428.

Estrada-Perez, C. \& Hassan, Y., 2010. PTV experiments of subcooled boiling flow through a vertical rectangular channel. International Journal of Multiphase Flow, 36(9), pp.691-706.

Estrada-Perez, C.E., 2004. Analysis, Comparison and Modification of Various Particle Image Velocimetry (PIV) Algorithms. USA, Tx: Texas A\&M University. 
Estrada-Perez, C.E. \& Hassan, Y.A., 2010. PTV experiments of subcooled boiling flow through a vertical rectangular channel. International Journal of Multiphase Flow, 36, pp.691-706.

Euh, D. et al., 2010. Characteristics of bubble departure frequency in a low-pressure subcooled boiling flow. Journal of nuclear science and technology, 47(7), pp.608-617.

Gabillet, C., Colin, C. \& Fabre, J., 2002. Experimental study of bubble injection in a turbulent boundary layer. International journal of multiphase flow, 28(4), pp.553-578.

Hassan, Y.A., Gutierrez-Torres, C.C. \& Jimenez-Bernal, J.A., 2005. Temporal correlation modification by microbubbles injection in a channel flow. Int. Com. Heat Mass Transfer, 32(8), pp.1009-1015.

Klausner, J. et al., 1993. Vapor bubble departure in forced convection boiling. International journal of heat and mass transfer, 36(3), pp.651-662.

Končar, B., Kljenak, I. \& Mavko, B., 2004. Modelling of local two-phase flow parameters in upward subcooled flow boiling at low pressure. Int. J. Heat Mass Transfer, 47, pp.1499-1513.

Koncar, B. \& Krepper, E., 2008. CFD simulation of convective flow boiling of refrigerant in a vertical annulus. Nuclear engineering and design, 238(3), pp.693-706.

Koncar, B., Krepper, E. \& Egorov, Y., 2005. CFD modelling of subcooled flow boiling for nuclear engineering applications. In International Conference "Nuclear Energy for New Europe ? Bled, Slovenia.

Koncar, B. \& Matkovic, M., 2012. Simulation of turbulent boiling flow in a vertical rectangular channel with one heated wall. Nuclear Engineering and Design, 245, pp.131-139.

Koncar, B. \& Tiselj, I., 2010. Influence of near-wall modelling on boiling flow simulation. Nuclear Engineering and Design, 240(2), pp.275-283.

Koyasu, M. et al., 2009. Turbulence structure of bubbly upward flow (high spatial and temporal resolution measurements using high speed time series PTV). Transactions of the Japan Society of Mechanical Engineers, 75 part B(755), pp.1446-1453.

Lee, T.H., Park, G.C. \& Lee, D.J., 2002a. Local flow characteristics of subcooled boiling flow of water in a vertical concentric annulus. Int. J. Heat Mass Transfer, 28(8), pp.1351-1368.

Lee, T.H., Park, G.C. \& Lee, D.J., 2002b. Local flow characteristics of subcooled boiling flow of water in a vertical concentric annulus. Int. J. Heat Mass Transfer, 28, pp.1351-1368.

Marie, J., Moursali, E. \& Tran-Cong, S., 1997. Similarity law and turbulence intensity profiles in a bubbly boundary layer at low void fractions. International journal of multiphase flow, 23(2), pp.227-247.

Mikielewicz, D., 2003. Hydrodynamics and heat transfer in bubbly flow in the turbulent boundary layer. International journal of heat and mass transfer, 46(2), pp.207-220.

Ortiz-Villafuerte, J. \& Hassan, Y.A., 2006. Investigation of microbubble boundary layer using particle tracking velocimetry. J. Fluid Mechanics, 128, p.507. 
Ramstorfer, F., Steiner, H. \& Brenn, G., 2008. Modeling of the microconvective contribution to wall heat transfer in subcooled boiling flow. Int. J. Heat Mass Transfer, 51, pp.4069-4082.

Ramstorfer, G. F. Steiner H. Brenn, 2007. Effect of the vapor phase on the near-wall velocity field in subcooled boiling flow. In Proccedings of the 6th International Conference on Multiphase Flow.

Roy, R.P. et al., 2002. Turbulent subcooled boiling flow experiments and simulations. J. Heat Transfer, 124, p.73.

Roy, R.P., Hasan, A. \& Kalra, S.P., 1993. Temperature and velocity fields in turbulent liquid flow adjacent to a bubbly boiling layer. Int. J. Multiphase Flow, 19, pp.765-795.

Roy, R.P., Velidandla, V. \& Kalra, S.P., 1997. Velocity field in turbulent subcooled boiling flow. J. Heat Transfer, 119, p.754.

Situ, R. et al., 2004. Flow structure of subcooled boiling flow in an internally heated annulus. Int. $J$. Heat Mass Transfer, 47(24), pp.5351-5364.

Thorncroft, G., Klausnera, J. \& Mei, R., 1998. An experimental investigation of bubble growth and detachment in vertical upflow and downflow boiling. International Journal of Heat and Mass Transfer, 41(23), pp.3857-3871.

Troshko, A. \& Hassan, Y., 2001a. A two-equation turbulence model of turbulent bubbly flows. International Journal of Multiphase Flow, 27(11), pp.1965-2000.

Troshko, A. \& Hassan, Y., 2001b. Law of the wall for two-phase turbulent boundary layers. International journal of heat and mass transfer, 44(4), pp.871-875.

Ünal, H., 1976. Maximum bubble diameter, maximum bubble-growth time and bubble-growth rate during the subcooled nucleate flow boiling of water up to $17.7 \mathrm{MN} / \mathrm{m}<\sup >2$. International Journal of Heat and Mass Transfer, 19(6), pp.643-649.

Wardana, I.N.G. et al., 1999. Turbulence structure in an annuli with strongly heated inner cylinder. Exp. Fluids, 27, pp.137-144.

Wardana, I.N.G., Ueda, T. \& Mizomoto, M., 1994. Effect of strong wall heating on turbulence statistics of a channel flow. Exp. Fluids, 18, pp.87-94.

Wardana, I.N.G., Ueda, T. \& Mizomoto, M., 1992. Structure of turbulent two-dimensional channel flow with strongly heated wall. Exp. Fluids, 13, pp.17-25.

Wardana, I.N.G., Ueda, T. \& Mizomoto, M., 1995. Velocity-temperature correlation in strongly heated channel flow. Exp. Fluids, 18, pp.454-461.

Yeoh, G.H. et al., 2002a. Prediction and measurement of local two-phase flow parameters in a boiling flow channel. Num. Heat Transfer, Part A, 42(1), pp.173-192.

Yeoh, G.H. et al., 2002b. Prediction and measurement of local two-phase flow parameters in a boiling flow channel. Num. Heat Transfer, Part A, 42, pp.173-192. 
Zhou, T. et al., 2013. Characteristics of a single bubble in subcooled boiling region of a narrow rectangular channel under natural circulation. Annals of Nuclear Energy, 57, pp.22-31. 